\title{
Sheehan Syndrome: a Rare Complication of Post-partum Hemorrhage
}

Soni M

Department of Obstetrics and Gynecology, SPMC and PBMH, Bikaner, Rajasthan, India

Received: February 8, 2013; Accepted: August 12, 2014

Incidence of Sheehan syndrome, also known as post-partum hypopituitarism or post-partum pituitary insufficiency or hypopituitarism syndrome has reduced markedly but it does occur in under-developed countries with a reported incidence of 1:10000 deliveries. ${ }^{1-3}$ We are reporting the case of a 22 year old female with Sheehan syndrome occurring after massive post-partum hemorrhage.

Keywords: hypopituitarism syndrome; post-partum hypopituitarism; post-partum pituitary insufficiency; Sheehan syndrome.

\section{INTRODUCTION}

Sheehan Syndrome is a condition that may occur in a woman who bleeds severely during childbirth. It implies hypopituitarism caused by necrosis of anterior pituitary gland due to blood loss and hypovolemic shock during and after childbirth. Such life-threatening hemorrhage has become uncommon as a result of the advances in obstetric care but it is still a major threat for women in developing countries. Consequences of post-partum hemorrhage can be severe as illustrated in this case report.

\section{CASE}

A 22-year old female, Mrs. S came with chief complaint of amenorrhea since her last delivery, three years back. She also complained of breast atrophy and decreased libido. She was a second para, with history of first uneventful delivery, uneventful postpartum period and breast feeding. During the second childbirth, which was conducted by a dai at home, she had heavy bleeding post-delivery followed by unconsciousness. She was taken to a hospital, where four units of blood were transfused. She was unable to breast-feed her baby, as "there was no breast milk."

\section{CORRESPONDENCE}

\section{Dr Monica Soni}

Department of Obstetrics and Gynecology, SPMC and PBMH, Bikaner, Rajasthan, India.

Email: drmonikasoni3008@gmail.com

Phone: +91-9982350350
She assumed this to be due to her weakness and did not consult any doctor.

On examination, she was a thin, pale lady, weighing $38 \mathrm{Kgs}$, with breast and genital atrophy and loss of axillary and pubic hair. Her pulse rate was 60/ minute and blood pressure was 90/50 $\mathrm{mm} \mathrm{Hg}$. Urine pregnancy test was negative. On per vaginum examination, the uterus felt smaller than normal size. An ultrasound was done, which showed a small uterus with very thin endometrium.

The patient was counseled for a hormonal workup. Her serum FSH, LH and estradiol values were low (FSH-2.20 mIU/ml, LH-1.00 $\mathrm{mIU} / \mathrm{ml}, \mathrm{E}_{2}-20$ $\mathrm{pg} / \mathrm{ml}$ ). These findings suggested that the cause for amenorrhea was at probably at pituitary level and owing to history of severe post-partum hemorrhage and lactation failure, Sheehan syndrome was suspected. Further work-up for confirmation showed low $\mathrm{TSH}, \mathrm{fT}_{4}$ and $\mathrm{fT}_{3}$, low morning serum cortisol and low growth hormone levels. These laboratory values suggested pituitary failure. MRI scanning of the pituitary was done and it showed empty sella and so the diagnosis of Sheehan syndrome was confirmed. The patient was started on cortisone, thyroxine and estrogen supplementation. After estrogen priming, progesterone was added. The patient was counseled that she may need to remain on hormone replacement therapy for rest of her lie with dose adjustments at the time of any surgery or other medical crisis. Initially 
the patient had hypomenorrhea but later the menstrual pattern improved and the general condition of the patient also improved.

\section{COMMENT}

Sheehan Syndrome, also known as Post-partum Hypopituitarism, Post-partum Pituitary Insufficiency or Hypopituitarism syndrome, ${ }^{1,2}$ is caused by anterior pituitary necrosis due to blood loss and hypovolemic shock during or after childbirth. Such life-threatening hemorrhage has become less common as a result of the advances in obstetric care but is still a major threat to women in developing countries. As such, Sheehan Syndrome is rare in industrialized nations but does occur in under-developed countries. The reported incidence is around 1:10,000 deliveries. ${ }^{3}$ The specific association of this syndrome with post-partum shock or hemorrhage was first described in 1937 by the British pathologist Harold Leeming Sheehan (19001988). ${ }^{2}$ Pituitary damage unrelated to pregnancy is called Simmond's Disease. ${ }^{2}$

Although the exact pathogenesis is still unclear, Sheehan Syndrome is believed to be the result of spasm in the arterial supply to the anterior lobe of the pituitary gland leading to ischemia and edema and ultimate necrosis and thrombosis in the portal sinuses and capillaries. ${ }^{2,3}$ Hypertrophy and hyperplasia of lactotrophs during pregnancy results in enlargement of the anterior pituitary without a corresponding increase in blood supply. ${ }^{2}$ Secondly, the anterior pituitary is supplied by a low pressure portal system. ${ }^{2}$ These vulnerabilities, when affected by a major hypotension or hemorrhage during the peri-partum period can result in ischemia of the affected pituitary regions leading to necrosis. ${ }^{2}$ The posterior pituitary is usually not affected due to its direct arterial supply by the inferior hypophyseal artery and the circle of Willis. ${ }^{2,3}$ A second theory as to the etiology is based on the development of Disseminated Intravascular Coagulation (i.e. in amniotic fluid embolism or HELLP syndrome) and intrapituitary bleeding. ${ }^{2,3}$

Any condition that increases the likelihood of severe blood loss during child-birth such as multiple pregnancies and abnormalities of the placenta increase the risk of Sheehan Syndrome. ${ }^{1,4}$ In most cases, the signs and symptoms appear slowly, after a period of months or years. ${ }^{4}$ The features are those of deficiencies of various hormones the pituitary gland controls: thyroid, adrenal, breast milk production and menstrual function. ${ }^{4}$ The presentation is highly variable. It is estimated that $95-99 \%$ of the anterior pituitary gland needs to be destroyed before the characteristic features appear. ${ }^{3}$ The most specific early postpartum sign is failure of lactation. ${ }^{3}$ Less extensive pituitary destruction (50-95\%) is associated with an atypical form of the disease with loss of one or more trophic hormones. ${ }^{3}$ The signs and symptoms of Sheehan Syndrome may include a galactorrhea (absence of lactation) and/or inability to breastfeed (breast milk never "comes in"), secondary amenorrhea, oligomenorrhea, genital and breast atrophy, hot flushes and decreased libido. ${ }^{1-3}$ Secondary hypothyroidism causes tiredness, intolerance to cold, constipation, weight gain, hair loss, slow thinking, slow heart rate and low blood pressure., ${ }^{2,3}$ Secondary adrenal insufficiency may manifest as loss of pubic and axillary hair, fatigue, weight loss, hypoglycemia, anemia and hyponatremia. ${ }^{2,3}$ Growth hormone deficiency causes many vague symptoms including fatigue and decreased muscle mass. ${ }^{2}$ The most serious complication is adrenal crisis, a sudden life-threatening state that can lead to extremely low blood pressure, shock, coma and death. ${ }^{4}$

Diagnosis is based on detailed history and examination of the patient combined with blood tests to measure the hormone levels. ${ }^{1}$ Dynamic provocative testing of the pituitary gland may be required. ${ }^{3}$ Low levels of TSH, ACTH, FSH and LH with low levels of $\mathrm{T}_{4}$, cortisol and estradiol are reported. Low level of IGF-1 suggests GH deficiency. Pituitary imaging with axial CT or MRI is indicated for diagnosis as well as to rule out other pituitary problems such as tumors. ${ }^{1}$ Treatment for Sheehan Syndrome is lifelong hormone replacement therapy. ${ }^{4}$ Any stress factor, even a minor one like influenza or common cold can cause a crisis and may require adjustment of these hormones. Treatment includes replacement of hydrocortisone first and then replacement of thyroid hormone and estrogen with or without progesterone, depending on whether the patient has a uterus or not. Hydrocortisone is replaced first because thyroxine therapy can exacerbate glucocorticoid deficiency and induce adrenal crisis. Replacement of growth hormone is controversial. 
In summary, the purpose of this case report is to create awareness among physicians that Sheehan syndrome is uncommon as a result of improved obstetric care but it should be a consideration in any woman who has a history of post-partum hemorrhage and reports signs and symptoms of pituitary deficiency.

\section{DISCLOSURE}

The authors report no conflicts of interest in this work. No violation of human rights and safety.

Funding: Nil

\section{REFERENCES}

1. Sheehan syndrome- PubMed Health. [Cited 2014 November 1]. Available from http://www.ncbi.nlm.nih.gov

2. Sheehan's syndrome- Wikipedia, the free encyclopedia. [Cited 2014 November 12]. Available http://www. en.wikipedia.org/ wiki/Sheehan's syndrome
3. Belfort MA. Thyroid and Other Endocrine Emergencies, In: Foley MR, Strong TH Jr, Garite TJ, editors. Obstetric Intensive Care Manual. $3^{\text {rd }}$ ed. New Delhi, India: Tata McGraw Hill; 2011.p.131.

4. Sheehan syndrome. [Cited 2014 December 1]. Available from http://www.cnn.com/HEALTH/library/sheehans-syndrome/ DS00889 